\title{
Decay Spectroscopy of Highly Charged Radioactive Ions with TITAN at TRIUMF
}

\section{Foster}

Department of Physics, University of Surrey, Guildford, GU2 7XH, United Kingdom TRIUMF, 4004 Wesbrook Mall, Vancouver, BC, V6T 2A3, Canada

\section{K.G. Leach*}

Department of Physics, Colorado School of Mines, Golden, CO, 80401, USA

E-mail: kleach@mines.edu

\section{Dillmann, C. Babcock, M. Good, A.A. Kwiatkowski, A. Lennarz}

TRIUMF, 4004 Wesbrook Mall, Vancouver, BC, V6T 2A3, Canada

\section{J. Dilling, E. Leistenschneider}

Department of Physics and Astronomy, UBC, Vancouver, BC, V6T 1Z1, Canada

TRIUMF, 4004 Wesbrook Mall, Vancouver, BC, V6T 2A3, Canada

\section{R. Klawitter}

Max-Planck-Institut für Kernphysik, Saupfercheckweg, D-69117 Heidelberg, Germany

TRIUMF, 4004 Wesbrook Mall, Vancouver, BC, V6T 2A3, Canada

\section{Andreoiu}

Department of Chemistry, Simon Fraser University, Burnaby, BC V5A 1S6, Canada

\section{T. Brunner}

Department of Physics, McGill University, Montreal, QC H3A 2T8, Canada

TRIUMF, 4004 Wesbrook Mall, Vancouver, BC, V6T 2A3, Canada

\section{Frekers}

Institut für Kernphysik, Westfalische Wilhelms-Universität, D-48149 Münster, Germany

For modes of electroweak decay such as electron capture and internal conversion, the spatial distribution of the electron cloud of the atom plays a major role in the decay probability. By successively removing electrons from these systems the decay probability is altered, thus changing the transition rates. In some cases, this can increase or decrease the decay rates by several orders of magnitude. These effects are particularily important in hot, ionizing, astrophysical environments. Due to significant technical challenges of generating and storing highly charged ions (HCIs), studies on these decay modes for HCIs are rare. With the commissioning of the TITAN decay spectroscopy setup at TRIUMF, nuclear decay properties of HCIs can now be studied in ion-traps for the first time. This proceeding reports the technical upgrade of the high-voltage infrastructure to provide access to increasingly ionized radioactive ions for decay study.

The 26th International Nuclear Physics Conference

11-16 September, 2016

Adelaide, Australia

\footnotetext{
*Speaker.
} 

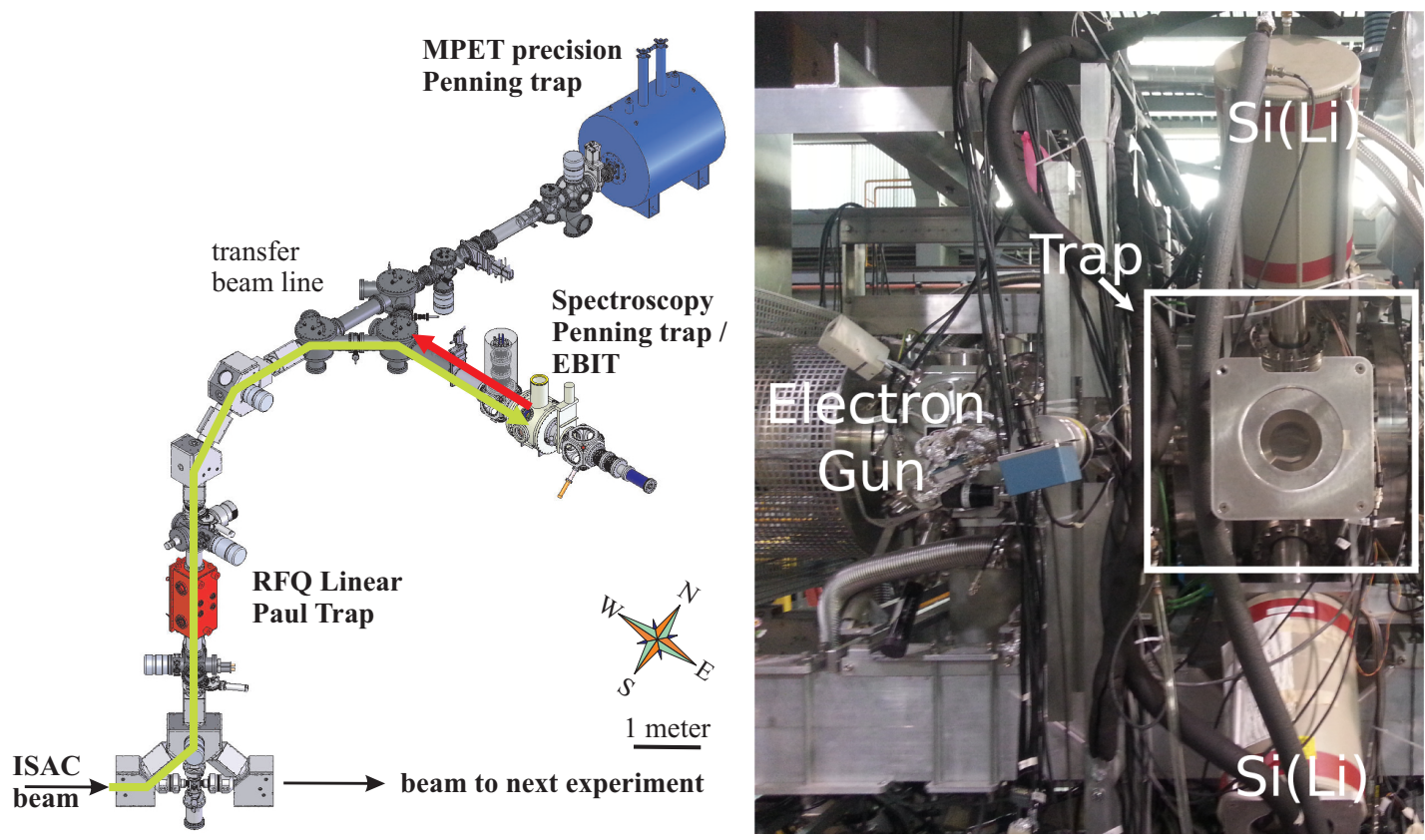

Figure 1: (left) A schematic of the current TITAN experimental setup in the ISAC facility at TRIUMF. The injection of bunched, singly charged ions into the EBIT is displayed by the green line, while the extraction of HCIs is shown by the red line. (right) A photograph of the EBIT decay spectroscopy setup in its configuration on the TITAN platform.

\section{Introduction}

Most forms of nuclear decay involve only the bound protons and neutrons that constitute the atomic nucleus, and require little-to-no interactions with the electrons that typically surround them. However, some common modes of electroweak decay such as orbital electron capture (EC) and internal electron conversion (IC), proceed through an interaction between the nucleus and bound electrons within the constituent atom. Additionally, for radioactive decay modes that emit charged leptons ( $\beta^{+} / \beta^{-}$decay which emit positrons/electrons, respectively), interactions with the surrounding electron cloud of the atom can change the energy and shape of the observed particle-emission momentum distributions. As a result, these respective decay modes are not only influenced by the structure of the initial and final states in the nucleus, but can also depend strongly on the atomic charge state [1]. These effects, particularly for EC and IC, become increasingly more significant as the atom is ionized closer to electron shells with the largest spatial overlap with the nucleus ( $K$ and $L$ shell).

In extreme cosmic environments, such as the acceleration of material by supernovae ( $\mathrm{SN}$ ) explosions to relativistic energies ( $>200 \mathrm{MeV} / u$ ) of galactic cosmic-ray particles, even heavy atomic systems with large numbers of bound electrons can be fully ionized. Several measurements of decay properties for these systems have been performed under terrestrial conditions, mainly via $\beta^{ \pm}$- or $\gamma$-spectroscopy, but very few (or none) have been performed under the proper astrophysical conditions. Under those conditions, the EC lifetimes do not become significantly affected until the atom is nearly fully stripped of its bound electrons. In fact, in most cases, orbital EC is only 

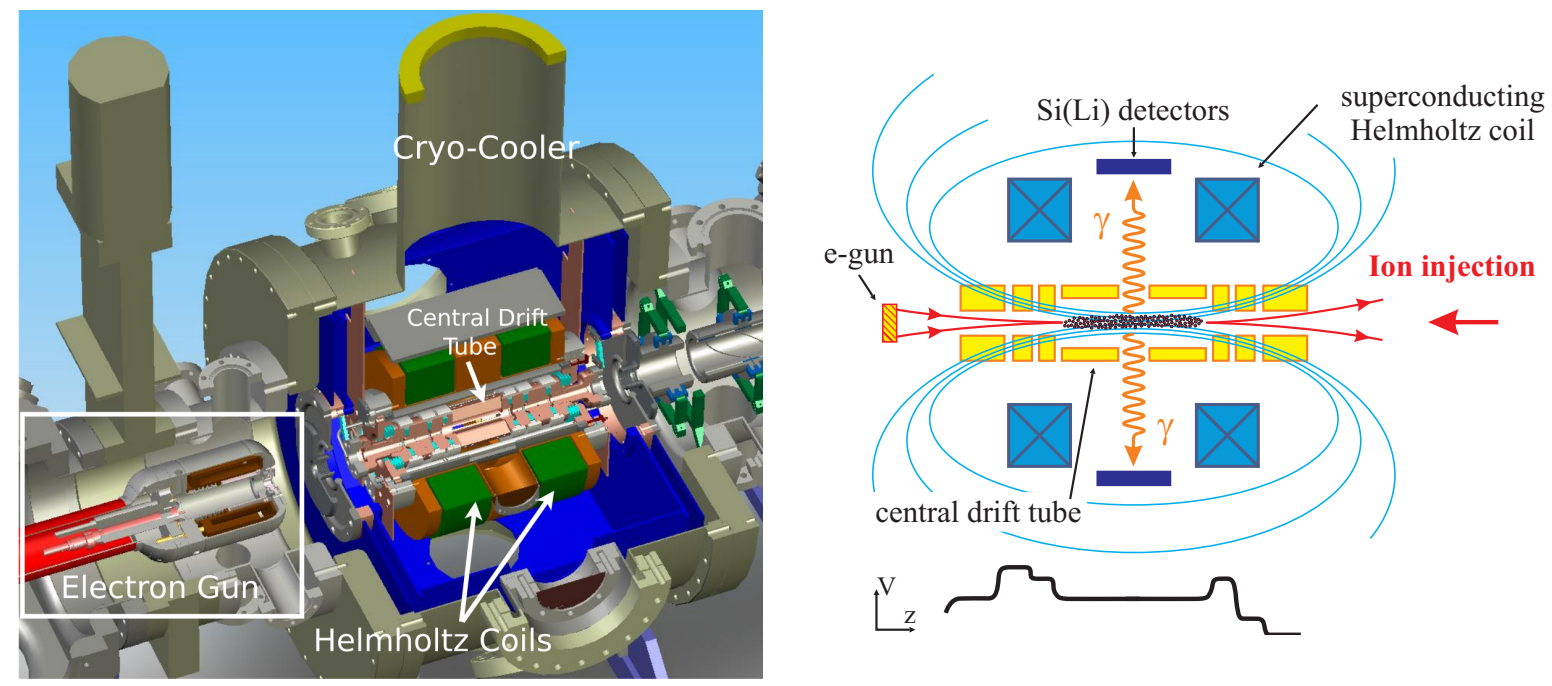

Figure 2: (left) A schematic view of the trap and electron-gun portions of the TITAN EBIT. (right) An illustration of the EBIT operation for decay spectroscopy and charge breeding (not to scale). Depicted below the trap components is a typical potential profile of the trap electrodes while the ion bunch is confined in the EBIT.

strongly influenced by the density of electrons in the $K$ and $L$ atomic shells. In order to provide the required environment in the laboratory for these studies, higher electron-beam energies are required to provide ionization of these heavier nuclei to He-like, H-like, and bare configurations.

For several years there has been interest in trying to perform measurements of highly charged ions (HCIs) in ion traps at low energies due to the increased experimental control over the decay environment. This article reports the progress of such work using the only low-energy ion trap in the world capable of performing experiments on the decay of HCIs.

\section{The TITAN Facility at TRIUMF-ISAC}

Due to the typically short decay half-lives of the radioactive nuclides of interest $\left(T_{1 / 2} \approx 10^{-3}\right.$ $10^{3} \mathrm{~s}$ ), they must be produced, purified, and delivered to the respective experimental setups in a short amount of time. The Isotope Separator and ACcelerator (ISAC) facility [2] at TRIUMF in Vancouver, Canada, employs a high-intensity (up to $100 \mu \mathrm{A}$ ) beam of $500 \mathrm{MeV}$ protons to produce radioactive ion beams (RIBs) using the isotope separation on-line (ISOL) technique [3]. The massselected continuous beam of radioactive singly charged ions (SCIs) is delivered at low energies $(<60 \mathrm{keV})$ to TRIUMF's Ion Trap for Atomic and Nuclear Science (TITAN) [4], which consists of three ion traps:

1. A radio-frequency quadrupole (RFQ) linear Paul trap [5] for buffer-gas cooling and bunching the low-energy ion beam,

2. A 3.7 T, high-precision mass-measurement Penning trap (MPET) [6], and

3. An electron-beam ion trap (EBIT) which is used to create HCIs [7], and for performing decay spectroscopy on trapped radioactive nuclei [8]. 


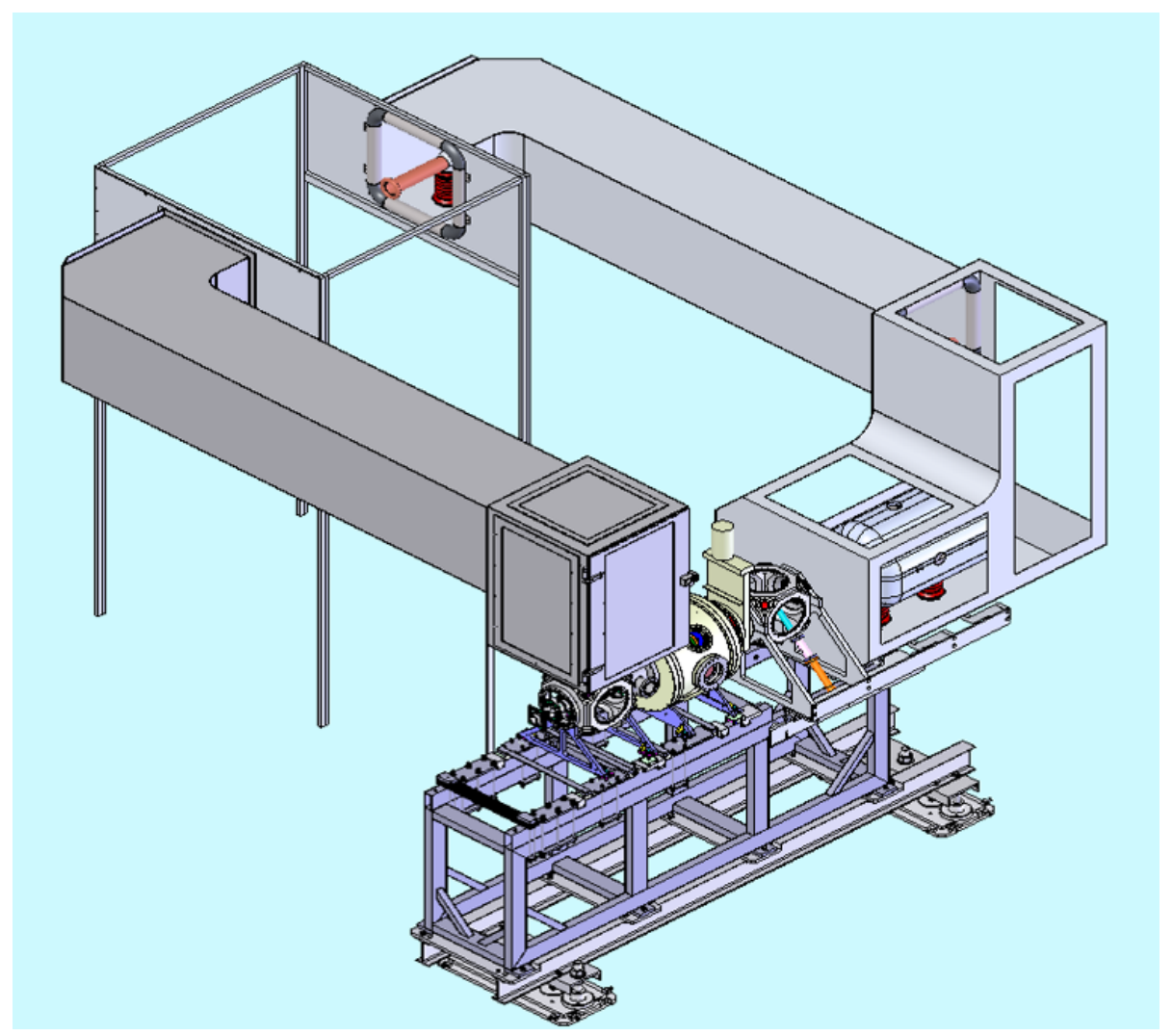

Figure 3: A design drawing of the HV upgrade enclosure for the TITAN EBIT to allow for $65 \mathrm{kV}$ operation.

A schematic view of the TITAN facility at TRIUMF-ISAC is shown in Fig. 1, along with the ion path for typical decay-spectroscopy experiments.

\section{The TITAN EBIT}

The TITAN EBIT, shown in Fig. 2 generates HCIs through the process of electron-impact ionization. The trap itself consists of an electron gun, a cold drift-tube assembly which is thermally coupled to a superconducting magnet, and an electron collector. The trapped ions are axially confined by an electrostatic square-well potential formed by applying voltage to the drift tubes. The typical voltage profile employed for trapping and breeding the ions is shown in the lower part of Fig. 2 (right). Radial confinement is provided by both the electron-beam space-charge potential, and an up-to 6 Tesla magnetic field produced by two superconducting coils in a Helmholtz-like configuration [7].

The EBIT features seven external ports separated by $45^{\circ}$ from each other, each with a $35 \mathrm{~mm}$ radius opening that is separated from the vacuum of the trap by $0.25 \mathrm{~mm}$ thick beryllium windows. For performing decay-spectroscopy experiments on HCIs, these ports can house individual photon detectors that allow for high-sensitivity characterization of radioactive decay [8]. Additionally, the in-trap composition of ion species and charge states can be periodically monitored by pulsing the trap contents out to a micro-channel plate (MCP) detector that is located downstream of the EBIT 

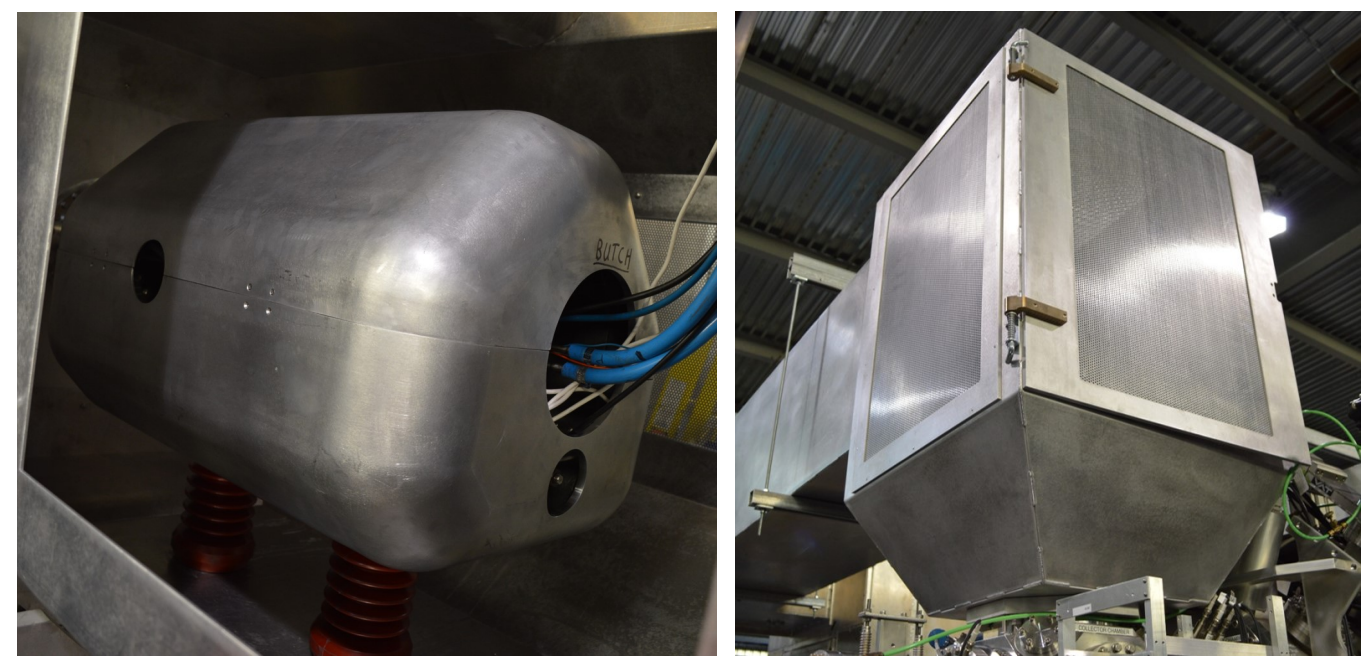

Figure 4: Images of (left) the electron gun enclosure and (right) electron-collector lockout cage.

in the TITAN beamline. The EBIT is operated in a cycled mode which typically consists of three parts: injection, storage/trapping, and extraction. The cycles are optimized to increase the photon signal-to-background ratio from the atomic transitions in the species of interest, and thus trapping portions of the cycle can last anywhere from a few seconds to minutes.

Two commissioning measurements with the current setup have already been performed: ${ }^{124} \mathrm{Cs}$ EC decay and ${ }^{116}$ In IC decay, which are reported in Refs. [9] and [10], respectively. For these experiments, charge states of $q=28^{+}$and $q=22^{+}$were used, corresponding to stripping electrons near the respective atomic $N$ shells. Despite the relatively large number of electrons that remained, atomic effects on the order of $\sim 100 \mathrm{eV}$ were observed, and demonstrated the high-level of sensitivity achievable with this setup. These relatively low ionization levels of the trapped atoms were sufficient for the commissioning, but must be improved to achieve the physics goals of this program. Currently, the TITAN EBIT utilizes an up-to $500 \mathrm{~mA}, 2 \mathrm{keV}$ electron beam.

\section{TITAN EBIT HV Upgrade}

To date, the TITAN EBIT maximum electron energy has been limited to a maximum of $7 \mathrm{keV}$. With the recent development of the HCI decay program outlined above, an upgrade of the EBIT $\mathrm{HV}$ infrastructure was required for investigations into decay rate modifications. With an operation voltage of $65 \mathrm{kV}$, access to bare ions up to $\mathrm{Z} \sim 70$ will now be possible.

In order to operate the system at $65 \mathrm{kV}$, three primary improvements to the system were required:

1. Internal sharp edges be rounded to a minimum radius of $30 \mathrm{~mm}$, and a minimum separation allowance of $300 \mathrm{~V} / \mathrm{mm}$ between grounded and biased surfaces be maintained to reduce the electric fields. 


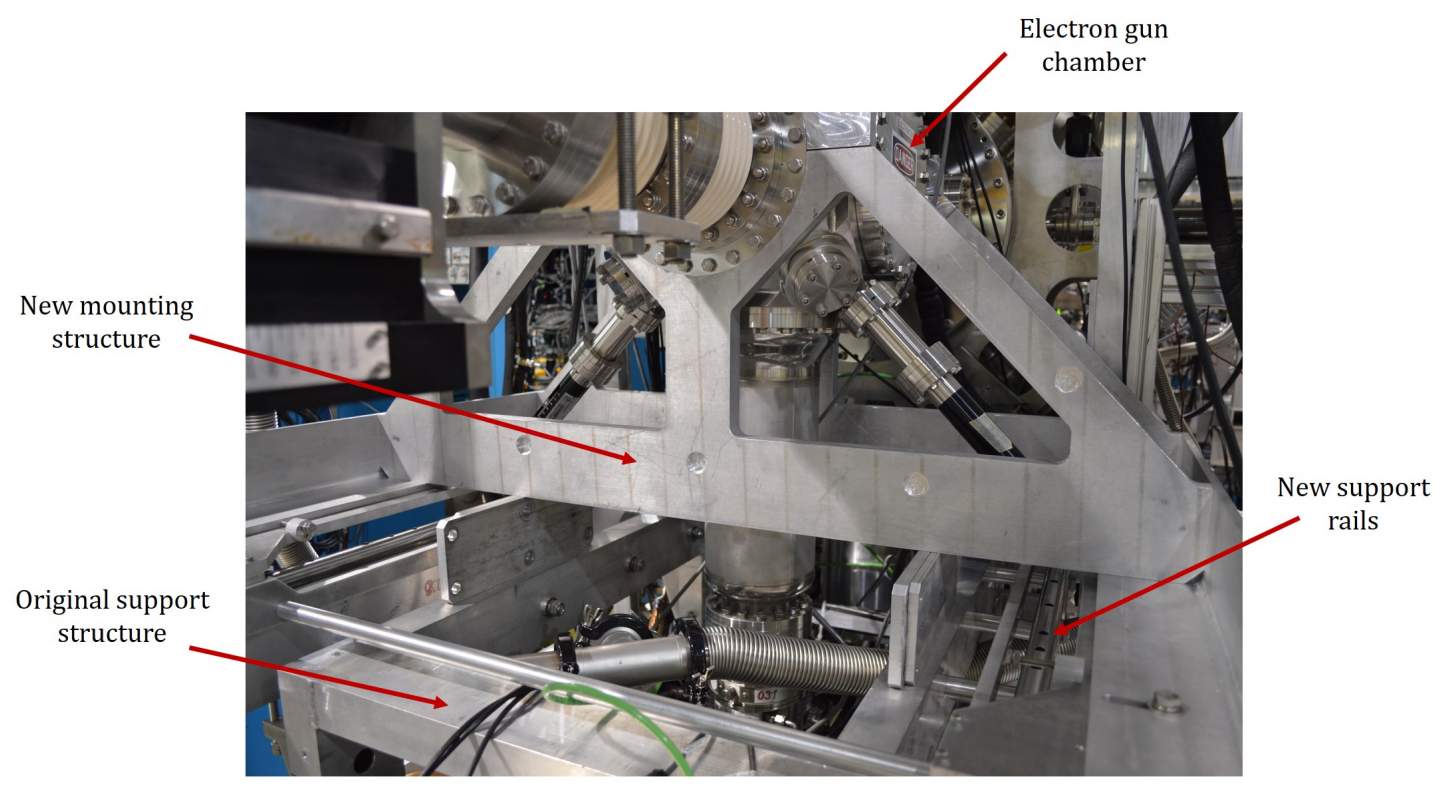

Figure 5: Installation of new support rails on which the electron gun, chamber and enclosure are mounted.

2. Access to high voltage components be restricted to prevent personnel entry before the risks have been minimised.

3. Overhead access to the trap and detectors not to be obstructed.

Using the above constraints on the design, the new infrastructure for safe operation at $65 \mathrm{kV}$ is displayed in Fig. 3. Based on the required separation allowance of $300 \mathrm{~V} / \mathrm{mm}$, the minimum separation distance between ground and any surface biased to $65 \mathrm{kV}$ is $0.217 \mathrm{~m}$. Due to the increased field strength at sharp edges, all edges within a high voltage enclosure must be rounded to a minimum radius of $30 \mathrm{~mm}$. The upgrade therefore features corona guards at pipe and duct ends, and rounded endcaps that secure the pipes to the insulators. The complexity of the fields at the electron gun manipulator required the entire manipulator to be housed in its own enclosure. Additionally, the electron collector required a lockout cage for $65 \mathrm{kV}$ operation. Images of these safety enclosures are shown in Fig. 4.

\section{Conclusions}

In summary, the TITAN EBIT decay spectroscopy setup provides a unique environment for probing electroweak decay properties using HCIs. The current experimental program is based on studying EC in hot astrophysical environments, as well as rare second-order decay processes to probe fundamental nuclear structure observables. The TITAN facility is currently undergoing multiple improvements to the experimental system [11]. The infrastructure for this upgrade at TITAN has been completed, and a new electron gun is currently being constructed at the Max-Planck Institute for Nuclear Physics in Heidelberg, and will be delivered in 2017. The full installation of the 
upgrade of the TITAN EBIT high voltage operation was completed in mid-December 2016, and will be commissioned with stable beam in the first half of 2017 .

\section{Acknowledgements}

The authors would like to thank the TITAN collaboration and TRIUMF machine shop for their efforts towards the development of this program. This work is supported in part by the National Science and Engineering Research Council of Canada (NSERC), the Deutsche Forschungsgemeinschaft (DFG) under grant FR 601/3-1, and Brazil's Conselho Nacional de Desenvolvimento Científico e Tecnológico (CNPq). TRIUMF receives federal funding via a contribution agreement with the National Research Council of Canada (NRC).

\section{References}

[1] Y.A. Litvinov and F. Bosch, Rep. Prog. Phys. 74, 016301 (2011).

[2] P.G. Bricault, M. Dombsky, P.W. Schmor, and G. Stanford, Nucl. Instrum. Meth. Phys. Res. B 126, 231 (1997).

[3] Y. Blumenfeld, T. Nilsson, and P. Van Duppen, Phys. Scr. T152, 014023 (2013).

[4] J. Dilling, R. Baartman, P. Bricault, M. Brodeur, L. Blomeley, F. Buchinger, J. Crawford, J.R. Crespo López-Urrutia, P. Delheij, M. Froese, G.P. Gwinner, Z. Ke, J.K.P. Lee, R.B. Moore, V. Ryjkov, G. Sikler, M. Smith, J. Ullrich, and J. Vaz, Int. Journ. Mass Spec. 251, 198 (2006).

[5] T. Brunner, M.J. Smith, M. Brodeur, S. Ettenauer, A.T. Gallant, V.V. Simon, A. Chaudhuri, A. Lapierre, E. Mané, R. Ringle, M.C. Simon, J.A. Vaz, P. Delheij, M. Good, M.R. Pearson, and J. Dilling, Nucl. Instrum. Methods Phys. Res. A 676, 32 (2012).

[6] M. Brodeur, V.L. Ryjkov, T. Brunner, S. Ettenauer, A.T. Gallant, V.V. Simon, M.J. Smith, A. Lapierre, R. Ringle, P. Delheij, M. Good, D. Lunney, and J. Dilling, Int. Journ. Mass Spec. 310, 20 (2012).

[7] A. Lapierre, M. Brodeur, T. Brunner, S. Ettenauer, A.T. Gallant, V. Simon, M. Good, M.W. Froese, J.R. Crespo López-Urrutia, P. Delheij, S. Epp, R. Ringle, S. Schwarz, J. Ullrich, and J. Dilling, Nucl. Instrum. Methods Phys. Res. A 624, 54 (2010).

[8] K.G. Leach, A. Grossheim, A. Lennarz, T. Brunner, J.R. Crespo López-Urrutia, A.T. Gallant, M. Good, R. Klawitter, A.A. Kwiatkowski, T. Ma, T.D. Macdonald, S. Seeraji, M.C. Simon, C. Andreoiu, J. Dilling, and D. Frekers, Nucl. Instrum. Meth. Phys. Res. A 780, 91 (2015).

[9] A. Lennarz, A. Grossheim, K.G. Leach, M. Alanssari, T. Brunner, A. Chaudhuri, U. Chowdhury, J.R. Crespo López-Urrutia, A.T. Gallant, M. Holl, A.A. Kwiatkowski, J. Lassens, T.D. Macdonald, B.E. Schultz, S. Seeraji, M.C. Simon, C. Andreoiu, J. Dilling, and D. Frekers, Phys. Rev. Lett. 113, 082502 (2014).

[10] K.G. Leach, A. Lennarz, A. Grossheim, R. Klawitter, T. Brunner, A. Chaudhuri, U. Chowdhury, J.R. Crespo López-Urrutia, A.T. Gallant, A.A. Kwiatkowski, T.D. Macdonald, B.E. Schultz, S. Seeraji, C. Andreoiu, D. Frekers, and J. Dilling, JPS Conf. Proc. 6, 020040 (2015).

[11] K.G. Leach, A. Lennarz, A. Grossheim, C. Andreoiu, J. Dilling, D. Frekers, M. Good, and S. Seeraji, EPJ Web of Conferences 93, 07006 (2015). 